Staff Working Paper/Document de travail du personnel 2021-4

Last updated: January 21, 2021

\title{
Networking the Yield Curve: Implications for Monetary Policy
}

by Tatjana Dahlhaus, ${ }^{1}$ Julia Schaumburg ${ }^{2}$ and Tatevik Sekhposyan ${ }^{3}$

${ }^{1}$ Canadian Economic Analysis Department

Bank of Canada, Ottawa, Ontario, Canada K1A 0G9

${ }^{2}$ Department of Econometrics and Data Science

Vrije Universiteit Amsterdam, 1081 HV Amsterdam, The Netherlands

${ }^{3}$ Department of Economics

Texas A\&M University, Texas 77843, USA

${ }^{3}$ Economic Research Department

Federal Reserve Bank of San Francisco, San Francisco, CA 94105, USA

dahl@bankofcanada.ca, j.schaumburg@vu.nl, tsekhposyan@tamu.edu

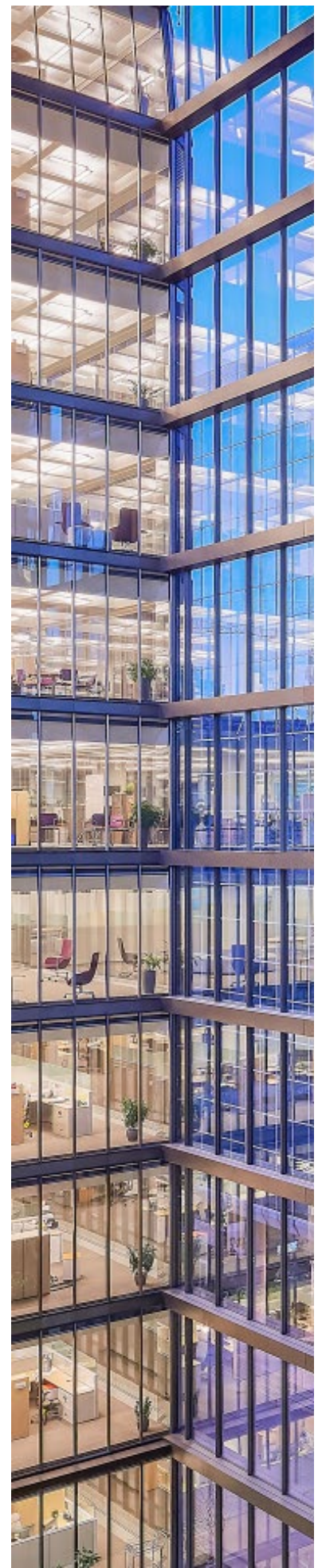

Bank of Canada staff working papers provide a forum for staff to publish work-in-progress research independently from the Bank's Governing Council. This research may support or challenge prevailing policy orthodoxy. Therefore, the views expressed in this paper are solely those of the authors and may differ from official Bank of Canada views. No responsibility for them should be attributed to the Bank. 


\section{Acknowledgements}

We thank Ivan Petrella, Ray Walker, the participants of the 2020 EABCN Conference on Empirical Advances in Monetary Policy; 2020 Real-Time Data Analysis, Methods, and Applications Conference; 2020 Econometric Society World Congress; and 2019 Computational and Financial Econometrics conference, as well as the seminar participants at the Bank of Canada for comments. We thank Refet Gürkaynak for kindly sharing data relevant for constructing monetary policy factors. This paper has been prepared under the Lamfalussy Fellowship Program sponsored by the European Central Bank (ECB). All views are those of the authors and do not necessarily represent the views of the ECB, the Eurosystem, the Bank of

Canada, the Federal Reserve Bank of San Francisco or the Board of Governors of the Federal Reserve System. 


\section{Abstract}

We introduce a flexible, time-varying network model to trace the propagation of interest rate surprises across different maturities. First, we develop a novel econometric framework that allows for unknown, potentially asymmetric contemporaneous spillovers across panel units and establish the finite sample properties of the model via simulations. Second, we employ this innovative framework to jointly model the dynamics of interest rate surprises and to assess how various monetary policy actions-for example, short-term, long-term interest rate targeting and forward guidance-propagate across the yield curve. We find that the network of interest rate surprises is indeed asymmetric and defined by spillovers between adjacent maturities. Spillover intensity is high on average but shows strong time variation. Forward guidance is an important driver of the spillover intensity. Pass-through from short-term interest rate surprises to longer maturities is muted, yet there are stronger spillovers associated with surprises at medium- and long-term maturities. We illustrate how our proposed framework helps our understanding of the ways various dimensions of monetary policy propagate through the yield curve and interact with each other.

Bank topics: Econometric and statistical methods; Monetary policy implementation; Interest rates

JEL codes: C18, C21, C53, E43, E44, E52 


\section{Introduction}

In the past few decades, the nature of monetary policy-making has changed. In addition to the traditional approach of using the short-term policy rate to stabilize the economy, central banks across the globe have increasingly relied on unconventional policy tools - targeting yields of different maturities has been at the forefront of policy-making. Following the financial crisis, central banks affected longer-term interest rates through large-scale asset purchases and forward guidance. Lately, yield curve control, i.e., direct targeting of yields at different maturities, has gained traction as a policy tool amid COVID-19. For example, the Reserve Bank of Australia adopted a form of yield curve control in March 2020 and targets the three-year government bond yield at $0.25 \%$.

Motivated by the changing policy landscape, we propose a novel framework that jointly models the various dimensions of monetary policy. We consider three possible dimensions: (i) conventional monetary policy operating through short-term interest rate targeting; (ii) unconventional monetary policy operating through the slope of the yield curve, which could be due to various forms of quantitative easing programs or direct yield curve control, and (iii) forward guidance - communication from the Federal Reserve about the state of the economy and likely path of monetary policy.

More specifically, we jointly model monthly interest rate surprises at various maturities (short, medium, and long ends of the yield curve) via a time-varying network, where spillovers can happen contemporaneously. This approach enables us to simultaneously study how monetary policy surprises (e.g., deviations from expectations in the three-months Treasury bills but also in the 10-year or three-year Treasury bond yields) propagate across different maturities. In addition, in our framework, forward guidance, as well as overall market conditions, can determine the intensity/interconnectedness of the network. Thus, the implied propagation of forward guidance and market conditions is time-varying.

Our model has two important features: the contemporaneous relationships between surprises at various maturities are allowed to be asymmetric and time-varying. In particular, we introduce a dynamic spatial lag model, where the network structure capturing the contemporaneous relationships between the variables can be characterized by an unknown, potentially asymmetric "weights" matrix estimated from the data. The intensity of the network, i.e., the strength of spillovers, is time-varying and may depend on covariates. While in our empirical specification we focus on interest rate surprises, the proposed empirical framework is general and can be used to study time-varying spillovers across any panel data set with moderate cross-sectional dimension (subject to identification restrictions discussed further). It is especially well suited for macroeconomic time series where 
the time-varying degree of simultaneity is of particular interest.

The contribution of the paper is twofold. First, we contribute to the econometric literature by proposing an extension of the spatial lag model that allows for asymmetric and unknown weights capturing contemporaneous correlations. This is in contrast to most of the literature, where these weights are taken as given by, for example, economic or geographic distances (see, e.g., Anselin 1988 for textbook treatment of the issue). Our approach is more similar to Bhattacharjee and Jensen-Butler (2013), Bailey et al. (2016), Lam and Souza (2016) and Lam and Souza (2020), who estimate the weights. Yet these papers work under the assumption of sparseness of the weights matrix and do not consider timevarying spillovers. On the other hand, for example, Blasques et al. (2016), Catania and Billé (2017), and Wang et al. (2018) model time-varying networks in a generalized autoregressive score (GAS) framework, yet they assume the weights to be pre-determined. We expand on these papers and extend the GAS model to allow for an endogenous, potentially asymmetric weights matrix. Identification is achieved by including a set of unit-specific regressors. Further, the dynamics of the intensity parameter can be informed by covariates in addition to the score. This allows us to give economic interpretations to the observed network dynamics.

Second, we contribute to the extensive literature on monetary policy pass-through to interest rates of various maturities. For example, Kuttner (2001), Gürkaynak et al. (2005), and Campbell et al. (2012) model the impact of the various dimensions of monetary policy, such as short-term interest rate targeting, quantitative easing, as well as forward guidance, on the interest rates in a univariate setup. We instead model the unexpected movements in the interest rates (surprises) of various maturities jointly. Therefore, our paper is more in the vein of $\mathrm{Bu}$ et al. (2019) and Inoue and Rossi (2019), who define monetary policy more generally, bridging conventional and unconventional policies. Our approach differs in the sense that we model spillovers across different monetary polices - an issue gone unexplored so far. This allows us to provide novel and interesting insights about how the different dimensions of monetary policy interplay and potentially amplify or dampen each other.

To study the time-varying interest rate surprise spillovers at various maturities in the US, we rely on the Blue Chip Financial Forecasts (BCFF) survey. In particular, we obtain interest rate surprises as the forecast errors of interest rates for different maturities. The $\mathrm{BCFF}$ are particularly useful for the aim of this paper since they provide forecasts for maturities covering the whole yield curve.

We uncover an asymmetric network structure for the interest rate surprises, where direct spillovers between immediate neighbors are sufficient to characterize the spillovers across 
the yield curve. The estimated structure of the network can help us understand how unexpected deviations from the target on, for example, the three-year yield spill over to other maturities. Furthermore, the network displays considerable time variation, and forward guidance appears to be an important determinant of this observed time variation. More specifically, forward guidance, measured by the path factor extracted as in Gürkaynak et al. (2005), moves the network intensity. News about future monetary policy easings increase the strength of the network and intensify the contemporaneous correlations between the interest rate surprises of various maturities.

Our results, overall, suggest that pass-through from short-term monetary policy surprises to longer maturities is muted, while the pass-through associated with surprises at medium- and long-term maturities is stronger. This suggests that targeting higher maturity bond yields may be an useful approach to move all the yields in the same direction. Further, we consider a few monetary policy experiments, where we show how the various dimensions of monetary policy interact. More specifically, we consider idiosyncratic and simultaneous interventions in different segments of the bond market, while varying the strength of the forward guidance. We find that the overall effect on the yield curve depends on the size of monetary policy shocks, the targeted maturities, as well as the strength of forward guidance. Our study highlights the importance of the spillover structure for policy-makingdepending on policy objectives, it matters which maturities the central bank targets and by how much. This further demonstrates that careful consideration of complementarities of policy tools and their amplifications is important for policy design.

The paper proceeds as follows. Section 2 discusses the econometric framework. We demonstrate the validity of our estimation method and identification using Monte Carlo

simulations in Section 3. In Section 4, we apply the newly proposed method to interest rate surprises across the yield curve, discuss the evolving network structure, and link it to the US business cycle and forward guidance. Moreover, in Section 5, we assess how different dimensions of monetary policy propagate across maturities and how they interact with each other, showing spatial responses. Section 6 concludes.

\section{Dynamic Network Model with Unknown Weights}

To analyze the time-varying network structure of forecast errors across different maturities, we employ a dynamic version of the spatial lag model. We use a score-driven framework to model the dynamics, and we allow for an unknown asymmetric weights matrix. 


\subsection{The Dynamic Spatial Lag Model}

Let $y_{t}$ denote an $N$-dimensional vector of dependent variable observations at time $t$, i.e., $y_{t}=\left(y_{1 t}, \ldots, y_{N t}\right)^{\prime}$. In our case $y_{t}$ contains interest rate surprises for various maturities. In the spatial lag model, each entry $y_{i t}$ may depend on a $(K \times 1)$ vector of pre-determined variables $x_{i t}$, but also on the contemporaneous values of the other units $y_{j t}$, for $j \neq i$. The model is given by

$$
y_{t}=\rho_{t} W y_{t}+X_{t} \beta+e_{t} \quad e_{t} \sim N(0 ; \Sigma),
$$

where $\beta$ is a $(K \times 1)$-vector of common slope coefficients, and $X_{t}$ is the $(N \times K)$-matrix of regressors. $\rho_{t}$ is the scalar time-varying spatial dependence (or network intensity) coefficient. If $\rho_{t}=0$, there is no spatial dependence across the different elements of $y_{t}$. Further, $W$ is an unobserved, time-invariant, $(N \times N)$-network matrix of weights, $w_{i j}$, for $i, j=1, \ldots, N$, with rows restricted to add up to one and with zeros on the main diagonal $\left(w_{i i}=0\right)$. The distribution of the $N$-dimensional error term vector $e_{t}$ is assumed to be normal with zero mean and a diagonal covariance matrix $\Sigma,\left.1\right|^{2}$

Writing the model in its nonlinear reduced form

$$
y_{t}=Z_{t} X_{t} \beta+Z_{t} e_{t}
$$

with $Z_{t}=\left(I_{N}-\rho_{t} W\right)^{-1}$, which is assumed to exist, reveals that it can capture complex dynamic dependencies between shocks to the disturbances $e_{t}$ as well as the regressors $X_{t}$, as $Z_{t}$ is in general a full matrix. Using a power series expansion, it becomes clear how the model captures nonlinear feedback effects across units, i.e.,

$$
y_{t}=X_{t} \beta+\rho_{t} W X_{t} \beta+\rho_{t}^{2} W^{2} X_{t} \beta+\ldots+e_{t}+\rho_{t} W e_{t}+\rho_{t}^{2} W^{2} e_{t}+\ldots
$$

Therefore, spillovers from unit $i$ to other units $j$ depend on the respective weight in $W$ and the network intensity $\rho_{t}$. Feedback effects to unit $i$ itself occur if, for example, the weights $w_{i j}$ and $w_{j i}$ are non zero. Based on this equation one can easily obtain spatial responses reflecting the evolution of feedback in time $t$.

\footnotetext{
${ }^{1}$ Model 11 is an extension of the score-driven spatial model introduced by Blasques et al. (2016). In their paper, spillovers between sovereign credit default swap spreads are modeled using an observed network of spillovers between the financial sectors of eurozone countries, and the time-varying spatial dependence parameter serves as a measure of systemic risk. Here, in contrast, we relax the assumption that $W$ is observable and allow for a more general structure of $W$ (see Section 2.3).

${ }^{2}$ Note that it is straightforward to extend the model to non-Gaussian error terms. We work with the normal distribution as it is an appropriate choice for our application.
} 


\subsection{Dynamics of the Network Intensity}

To model the dynamics of the network intensity, $\rho_{t}$, we use the following reparameterization: $\rho_{t}=h\left(f_{t}\right)$, where $f_{t}$ is a time-varying parameter and $h\left(f_{t}\right)=\gamma \tanh \left(f_{t}\right)$ with $0<\gamma<1$ to ensure numerical stability ${ }^{3}$ We assume that $f_{t}$ follows an autoregressive score-driven dynamic process, which can be extended with a $(D \times 1)$-vector of exogenous regressors $r_{t}$, that is,

$$
f_{t+1}=d+a s_{t}+b f_{t}+r_{t}^{\prime} c
$$

where $a, b \in \mathbb{R}$ and $c \in \mathbb{R}^{D}$ are unknown coefficients. The intercept is restricted to $d=1-b$ for identification purposes. $s_{t}=S_{t} \nabla_{t}$ denotes the scaled score function at time $t$, and $S_{t}$ is a scaling factor ${ }^{4}$ The initial value $f_{0}$ is treated as an unknown parameter, which is estimated with our numerical optimization procedure. The score corresponds to the first derivative of the log-likelihood function at time $t$ with respect to $f_{t}$, i.e., $\nabla_{t}=\frac{\partial \ell_{t}}{\partial f_{t}}$.

The advantage of specifying the score as innovation term in the dynamics of $f_{t}$ is that the likelihood is available in closed form even for nonlinear models such as that in Equation (1). Score-driven models provide a general framework for modeling time variation in the parameters and were introduced in Creal et al. (2013) and Harvey (2013). It has been shown in Koopman et al. (2016) that in a wide variety of settings (in particular, when there are non-linearities and non-Gaussianity), these models perform as well as the more general but computationally much more intensive nonlinear state space models. ${ }^{5}$

The likelihood at time $t$ is given by

$$
\ell_{t}=\log \left|\left(\mathrm{I}_{N}-h\left(f_{t}\right) W\right)\right|-\frac{N}{2} \log (2 \pi)-\frac{1}{2} \log |\Sigma|-\frac{1}{2} e_{t}^{\prime} \Sigma^{-1} e_{t}
$$

The implied scaled score based on normally distributed errors is given by $s_{t}=S_{t} \nabla_{t}$ with

$$
\nabla_{t}=\left(y_{t}^{\prime} W^{\prime} \Sigma^{-1} e_{t}-\operatorname{trace}\left(Z_{t} W\right)\right) \dot{h}\left(f_{t}\right)
$$

where $e_{t}=\left(y_{t}-h\left(f_{t}\right) W y_{t}-X_{t} \beta\right), Z_{t}=\left(\mathrm{I}_{N}-h\left(f_{t}\right) W\right)^{-1}$ and $\dot{h}\left(f_{t}\right)=\frac{\partial h\left(f_{t}\right)}{\partial f_{t}}$. As discussed previously, we adopt unit scaling, i.e., $S_{t}=1$.

To estimate the unknown static parameters that are summarized in the vector $\theta$, we

\footnotetext{
${ }^{3}$ Note that $\gamma$ is not estimated but fixed. It is part of the reparameterization of $f_{t}$, in order to ensure that $\rho_{t}$ becomes numerically not too close to one during optimization $\left(\operatorname{since} \tanh \left(f_{t}\right) \approx 1\right.$ for large $\left.f_{t}\right)$. This would lead to an error in the evaluated likelihood. In the simulations and empirics, we set $\gamma=0.99$.

${ }^{4}$ We choose $S_{t}=1$. In the literature, $S_{t}$ is often chosen as the inverse of the Hessian. Since in our case this would involve integrating out the data-generating process, we proceed with the parsimonious choice of unity, similar to Blasques et al. (2016).

${ }^{5}$ For theory and empirics of different generalized autoregressive score (GAS) models, see also, e.g., Creal et al. (2011), Harvey and Luati (2014), Creal et al. (2014).
} 
numerically maximize the joint likelihood function

$$
L(\theta)=\sum_{t=1}^{T} \ell_{t},
$$

where $\theta=\left(w^{\prime}, a, b, c, f_{0}, \beta^{\prime}, \operatorname{diag}(\Sigma)^{\prime}\right)^{\prime}$, with $w$ being a vector containing all non-zero (offdiagonal) elements of $W$ and $\operatorname{diag}($.$) creating a vector of the diagonal elements of \Sigma$. Therefore, the likelihood function is evaluated simultaneously for the parameters entering the network intensity process as well as the network matrix.

\subsection{Unknown Weights Matrix}

Most of the literature assumes that the spatial weights matrix is pre-specified and symmetric, i.e., $w_{i j}=w_{j i}$. For example, $W$ could be defined by economic or geographic distances ${ }^{6}$ However, defining the weights matrix exogenously comes with a few well-known drawbacks: The choice of the weights can often seem arbitrary, there is substantial uncertainty regarding the choice, and empirical results may hinge on a specific choice of spatial weights. Here we deviate from the assumption of a pre-specified weights matrix and consider it as unknown. In addition, we relax the assumption of symmetry, as spillovers from unit $i$ to unit $j$ are not necessarily the same as from unit $j$ to unit $i$. These features are particularly useful when assessing the dependence between interest rate surprises across different maturities, since an obvious proxy for the network is not available. Also, estimating the weights matrix can provide insights into the drivers of economic interactions and general equilibrium effects in a network.

We assume that $W$ is a constant, asymmetric matrix of unknown parameters that describes the proximity of units $y_{i}$ and $y_{j}$ at each point in time $t$. $W$ has zeros on the main diagonal, and every other element is restricted to be non-negative. For interpretability, the weights in $W$ are assumed to lie between 0 and 1 , and we employ the standard practice of a row-normalized weights matrix, that is, $\sum_{j=1}^{N} w_{i j}=1$ for $i=1, \ldots, N$ where $w_{i j}$ is the $(i, j)$ th element of $W$. Row-normalization also restricts the largest eigenvalue of $W$ to equal one, which reduces the problem of ensuring stability to restricting the network intensity parameter to be smaller than one. In particular, we specify the elements of $W, w_{i j}$, using a multinomial transform,

$$
w_{i j}=\frac{\exp \left(-s_{i j}\right)}{\sum_{k=1, k \neq i}^{N} \exp \left(-s_{i k}\right)}, i \neq j, \quad \text { and } \quad w_{i i}=0,
$$

\footnotetext{
${ }^{6}$ For example, to model credit risk spillovers across international banks, banks' cross-border exposure based on debt data has been used as a proxy for the network structure. See, e.g., Blasques et al. (2016) and Tonzer (2015).
} 
where $s_{i j}$ are freely estimated economic distances between units.

\subsection{Identification}

It is essential to note that identification in our context is achieved by including unit-specific regressors $x_{i t}$; see Equation (1). It has been formally shown by Bhattacharjee and JensenButler (2013) that in a spatial lag model without regressors, a static, unknown spatial weights matrix $W$ is only identified for up to $N(N-1) / 2$ elements.7 To see that the remaining elements are identified in the presence of at least one unit-specific regressor, consider the reduced-form equation of the model without dynamic spatial dependence parameter and without normalization of $W$, i.e.,

$$
y_{t}=\left(\mathrm{I}_{N}-W\right)^{-1} X_{t} \beta+\left(\mathrm{I}_{N}-W\right)^{-1} e_{t} .
$$

For simplicity, suppose $K=1$, so $\beta$ is a scalar common slope parameter. In the equation for unit $i$, denoting the $i j$-th element of matrix $Z=\left(\mathrm{I}_{N}-W\right)^{-1}$ by $z_{i j}$, we have

$$
y_{i t}=\beta \sum_{j=1}^{N} z_{i j} x_{j t}+\sum_{j=1}^{N} z_{i j} e_{j t} .
$$

Since $\beta$ is pooled across the different regressor observations (and panel units), it is identified together with the $(N-1)$ coefficients $z_{i j}$, using the restrictions from the covariance matrix. The mapping from $Z$ to $W$ is unique, ensuring identification of the non-zero elements of $W$ as well. In general, pooling across the unit-specific regressors and panel units generates an additional $N^{2} K$ restrictions, which can be used for identification in conjunction with the $N(N-1) / 2$ restrictions in the covariance matrix.

Finally, in the presence of a dynamic spatial dependence parameter, the coefficients $\left(a, b, c, f_{0}\right)$ are identified off the Equation (4), provided the spatial weights matrix is normalized to have maximum eigenvalue one.

\section{Monte Carlo Study}

We assess the performance of the dynamic network model with an unknown asymmetric weights matrix in two settings. First, we investigate the performance of the model in filtering out different patterns of the network intensity parameter $\rho_{t}$. Second, we simulate from the true model to assess whether our model consistently estimates the unknown parameters

\footnotetext{
${ }^{7} \mathrm{~A}$ symmetric $W$ is fully identified in their setting. In our context, however, we want to allow for potential asymmetry as well.
} 
and, in particular, the elements of $W$.

In the first simulation experiment, the spatial dependence parameter $\rho_{t}$ is generated according to three deterministic patterns: a sine pattern,

$$
\rho_{t}=0.5+0.3 \cos (2 \pi \cdot t / 200)
$$

a step pattern with a break after half the sample size

$$
\rho_{t}=0.9-0.5(t>T / 2)
$$

and a constant, i.e., $\rho=0.9$.

The data-generating process is

$$
y_{t}=\left(\mathrm{I}_{N}-\rho_{t} W\right)^{-1}\left(X_{t} \beta+e_{t}\right) \quad e_{t} \sim N\left(\mathbf{0}, \sigma^{2} \mathrm{I}_{N}\right) .
$$

We use one unit-specific regressor $X_{t} \sim N\left(\mathbf{0}, 0.5 \cdot \mathrm{I}_{N}\right)$. The parameter values are $\beta=0.2$ and $\sigma^{2}=0.1$. Considered sample sizes are $T=300,600$ and $N=4,7$. The spatial weights matrix $W$ is generated in two stages: first, random draws from a normal distribution with mean 0.5 and standard deviation 1 are used for the off-diagonal elements and $w_{i i}=0$. Then, the matrix is standardized using a multinomial transform as in Equation (8), so that all elements are non-negative and the rows sum up to one.

We generate 250 samples using each combination of deterministic pattern, $T$, and $N$ and estimate our model. Table 1 reports results on mean absolute error for the filtered spatial dependence parameter $\widehat{\rho}_{t}$, its empirical 5\%-95\% quantile range, as well as mean squared errors for the average parameter estimates $\widehat{w}_{i j}, i, j=1, \ldots, N, \widehat{\beta}$ and $\widehat{\sigma}^{2}$. We observe that as we increase $T$, the tracking errors and range become smaller in all cases except for the step pattern and $N=4$. Generally, the static parameters are estimated more accurately for larger $T$ and $N$.

Figure 1, on the other hand, shows the true patterns of $\rho_{t}$ for $N=7$ and $T=300,600$, together with the filtered median paths and the corresponding $5 \%$ and $95 \%$ empirical quantiles across simulations 8 In case of the sine pattern, upward trends are captured more accurately than downward trends, but the true time-varying parameter always lies between the $5 \%$ and $95 \%$ quantiles. In case of the abrupt level decrease of $\rho_{t}$, it takes some observations for the filtered parameter to adapt. The empirical quantile range becomes larger after the downshift, possibly because the signal in the data has become weaker with the decreased spatial correlation. All in all, we conclude that the tracking performance is satisfactory.

\footnotetext{
${ }^{8}$ The plots for $N=4$ are extremely similar to the ones for $N=7$. They are available upon request.
} 
In the second experiment, we simulate from the true data-generating process to see how accurately the unknown parameters are estimated in case of practically relevant sample sizes. The model is

$$
\begin{aligned}
y_{t} & =\left(\mathrm{I}_{N}-h\left(f_{t}\right) W\right)^{-1}\left(X_{t} \beta+e_{t}\right) \quad e_{t} \sim N\left(0, \sigma^{2} \mathrm{I}_{N}\right), \\
f_{t+1} & =d+a s_{t}+b f_{t},
\end{aligned}
$$

where $s_{t}=S_{t} \nabla_{t}$ with $S_{t}=1$ and $\nabla_{t}$ is defined in Equation (6). We set $d=1-b$ for identification purposes and, as before, $x_{t} \sim N\left(0,0.5 \mathrm{I}_{N}\right)$. The parameter values are $a=0.04, b=0.9, f_{0}=1$ (set to its unconditional mean), $\beta=0.5$, and $\sigma^{2}=0.1$, and $W$ is constructed as in the previous simulation experiment. 250 samples of $y_{t}$ are simulated for $N=4,7,10$ cross-sections and $T=300,600,1000$ time points, respectively. Kernel densities for the case $N=4$ are shown in Figure 2, suggesting that all parameters are estimated consistently. Likewise, Table 2 documents that estimation accuracy, measured by mean squared error and mean absolute error, increases as sample sizes become larger, both in the cross-sectional and time dimension 9

From our simulations, we conclude that the new dynamic network model can be estimated reliably for sample sizes that are similar to those in our empirical application. Furthermore, all elements of the asymmetric spatial weights matrix $W$ are identified in the presence of unit-specific regressors.

\section{Networking the Yield Curve}

We now apply the proposed framework to model the time-varying contemporaneous spillovers of interest rate surprises across the yield curve. First, we discuss the data we use to measure interest rate surprises. We then present the estimated network structure and discuss the drivers of its time variation.

\subsection{Data}

We think of interest rate surprises as unexpected deviations from observed interest rates. In particular, to measure interest rate surprises of various maturities, we use forecast errors obtained from the BCFF survey. This survey is well suited for our purposes since it provides monthly forecasts of the whole yield curve. In particular, we use the forecasts of the threemonth Treasury bills (T-bills), six-month T-bills, and one-year, two-year, five-year, 10-year,

\footnotetext{
${ }^{9}$ We are not reporting the results for $f_{0}$, as it is a nuisance parameter. The results in Figure 1 indicate that $f_{0}$ is estimated correctly.
} 
and 30-year (long-run) Treasury notes to obtain forecast errors ${ }^{10}$ Forecasts of these interest rates are consistently available from February 1988 11

The BCFF survey is conducted monthly, covering approximately 50 analysts ranging from broker-dealers to economic consulting firms. The BCFF is published on the first day of each month and presents forecasts from a survey conducted during two consecutive business days one to two weeks earlier. The precise dates of the survey vary and are not generally noted in the publication. Each month the BCFF provides the forecasts of the average interest rate over a particular quarter, beginning with the current quarter and up to four or five quarters into the future. For example, in January, the forecast of the current quarter captures the average expected interest rate over January, February, and March, and the one-quarter-ahead forecast pertains to the average expected outcome of April, May, and June.

Therefore, the monthly BCFF forecasts are fixed-event forecasts of interest rates over the quarter, implying that their forecast horizon changes with each month in the quarter. We construct fixed-horizon forecasts by weighting the two given fixed-event forecasts following Chun (2011) (or see Dovern et al., 2012, for an application to survey forecasts of GDP and prices). In order to obtain, for instance, a six-months-ahead (fixed-horizon) forecast, we look at one-quarter- and two-quarters-ahead (fixed-event) forecasts. In the first month of the quarter, the six-months-ahead forecast is simply the forecast of the one-quarter-ahead forecast. In the second month of the quarter, the six-months-ahead forecast is obtained by taking the average of the one-quarter- and two-quarters-ahead forecasts with weights equal to $2 / 3$ and $1 / 3$, respectively. The six-months-ahead forecast for the final month of the quarter is the weighted average of the one-quarter- and two-quarters-ahead forecasts, with weights equal to $1 / 3$ and $2 / 3$. Nine-months-ahead forecasts are calculated as the weighted average of the two-quarters- and three-quarters-ahead forecasts given by the survey, with weights similar to the ones discussed above 12

\footnotetext{
${ }^{10}$ The US Treasury discontinued the 30-year Treasury constant maturity series in February 2002 and reintroduced it in February 2006. Over this period the BCFF provides forecasts for the long-term average ("LT>25") note from March 2002 to June 2004, and for the 20-year Treasury note from July 2004 to April 2006. We use these forecasts to fill in the period when the 30-year Treasury note was not available.

${ }^{11}$ For instance, the US Survey of Professional Forecasters (SPF) contains only forecasts for the threemonth Treasury bill and 10-year Treasury bond yields, starting from 1984:Q3 and 1992:Q1, respectively. Alternatively, one could use futures of US interest rates to proxy expectations. However, data coverage can be limited as some futures, such as Treasury-bill futures, are thinly traded. Further, futures data may include counterparty or liquidity risks.

${ }^{12}$ Alternatively, a researcher can use the methodologies proposed in Knüppel and Vladu (2016), where the fixed-event point forecasts are combined to yield the "best" fixed-horizon point forecasts in a mean squared forecast error sense. However, in our context, it is not obvious that the fixed-horizon forecasts should adhere to any optimality criterium. Moreover, Ganics et al. (2019) show that ad hoc weights are not noticeably inferior to the optimized weights, at least when looking at fixed-horizon density forecasts of GDP growth and inflation obtained from pooling the fixed-event ones.
} 
In our analysis, we use forecast errors at the six-months-ahead horizon 13 Finally, we obtain forecast errors by subtracting the consensus forecasts (mean across the 50 analysts) from the monthly realizations (calculated as average of daily figures) that are available from the Federal Reserve Board's H.15 website. The forecast errors for the interest rates at different maturities are plotted in Figure 3. We also use the lagged values of the first difference of the monthly realizations of the three- and six-month T-bills, one-, two-, five-, and 10-year Treasury notes, and 30-year (long-run) Treasury notes as exogenous regressors in our model (see Figure 4) 14 Finally, our sample period covers March 1988 until April 2016.

\subsection{Network of Interest Rate Surprises}

Using data of the interest rate surprises (forecast errors) at different maturities, we estimate the dynamic network model described in Section 3. To start with, we abstract from exogenous regressors in the dynamics of the spatial intensity parameter (Equation 4). First, we discuss the estimated weights matrix, followed by a discussion of the time-varying network intensity.

Figure 5 shows the estimated weights matrix (Panel a) and the corresponding network graph (Panel b) for the interest rate surprises at the seven different maturities. In the network graph, bigger and darker-colored nodes represent nodes with a larger number of connections. For instance, the one-year bond surprise is in a darker blue because it has five connections relative to the surprises in either the two-year, five-year, or 10-year maturities, where each has four connections. Edges have the same color as the node of origin. We can make two interesting observations.

First, Panel (a) shows that the estimated weights matrix and thus interconnectedness is asymmetric. This implies that, for example, the connectivity from six-month to one-year interest rate surprises differs from the connectivity of one-year to six-month surprises, and the latter is stronger. Moreover, the medium- to long-term surprises are more strongly connected relative to the surprises on the short end of the yield curve. Second, the table suggests that the estimated weights matrix is sparse - it mostly contains zeros below and above the main diagonal. Note that the only imposed zeros are on the main diagonal of the matrix; the rest are estimation outcomes. Looking at the network graph in Panel (b), we

\footnotetext{
${ }^{13}$ Also, the forecast errors are demeaned before entering the network model, i.e., Equation 1 In principle, one could think of using our empirical framework to model the interest rate surprises of a particular maturity across various forecast horizons or to model various maturities and forecast horizons jointly. We do not consider these scenarios here due to dimensionality concerns.

${ }^{14}$ For the period when the 30-year Treasury was not available, the US Treasury published a factor for adjusting the daily nominal 20-year constant maturity in order to estimate a 30-year nominal rate.
} 
can clearly see that the estimated network is defined by connectivity between immediate neighbors. That is, the surprises of three-month T-bill are connected to the surprises of six-month T-bill, while six-month T-bill surprises are connected to one-year surprises, oneyear surprises are connected to two-year surprises, and so on. Therefore, we do not find any evidence that short- and long-term interest rates surprises are directly connected.

We now turn to the time-varying network intensity. Figure 6 plots the spillover parameter, $\rho_{t}$, over time. Gray bars depict the NBER recession dates. Network intensity is high, on average, but shows strong time variation. The strength of spillovers seems to peak before recessions and remains high during these periods. Further, while the network intensity still exhibits strong time variation, it seems to decrease over the period when the federal funds rate reached zero lower bound (starting in December 2008). This is not surprising, as shorter-term surprises are relatively flat over that period, thus, potentially weakening the spatial correlations.

\subsection{Fundamentals as Drivers of Network Dynamics}

A natural question that follows is what drives the observed dynamics of the network intensity of yield curve surprises. In this section, we assume that the dynamics of the spillover intensity, $\rho_{t}$, may be affected by exogeneous regressors $\left(r_{t}\right.$ in Equation 4). Our maintained hypothesis is that forward guidance can affect the network dynamics - communicating the future path of interest rates has the potential to affect the correlation across various maturities simultaneously. Therefore, we model forward guidance to affect the intensity of spillovers. We also consider the business cycle and market uncertainty as additional drivers.

To proxy forward guidance, i.e., communication about the future path of the policy rate, we follow Gürkaynak et al. (2005) and use their "path" factor ${ }^{15}$ To assess the role of business cycle conditions, we use a monthly measure of economic growth, i.e., industrial production growth. Finally, we also include the VIX, the implied volatility measure from the S\&P 500 index options, as a determinant of the spillover intensity in our network model. The drivers are plotted in Figure 7 .

In Table 3, we present results for several specifications concerning the dynamics of the network intensity ${ }_{16}^{16}$ Model 1 refers to the case where the network intensity is driven by the score only. Model 2 refers to the specification where we also include the path factor as a potential driver. In Model 3, we add market uncertainty and, lastly, Model 4 includes all 3 regressors, that is, the path factor, the VIX, and IP growth. The table shows the estimated

\footnotetext{
${ }^{15}$ As an alternative, we could also use a text-based measure, similar to the one proposed in Gardner et al. (2020) to capture the forward guidance effects.

${ }^{10}$ Note that, in these cases, our sample starts in January 1990 due to data availability of the VIX.
} 
coefficients, i.e., vector $c$ from Equation (4) as well as $a$ and $b$ (for completeness). We also provide the Akaike information criteria (AIC) to compare the different model specifications.

Model 2, where the network intensity is driven by the path factor as well as the score, is the preferred model according to the AIC. Adding the VIX or economic growth as regressors does not improve the model. In turn, the estimated coefficients show that the path factor is negatively related to spillover intensity across all model specifications, implying that future lower interest rates are associated with a higher spillover intensity. This suggests that signaling future monetary easing increases the intensity of the network. Also, increasing market uncertainty and decreasing economic growth increase the spillover intensity. The estimated coefficients seem to provide one consistent story - network intensity increases due to higher uncertainty, which is generally associated with negative growth and future monetary policy expansions (to stimulate the economy).

To provide insights on the potential contributions of the three drivers, Figure 8 provides plots of the different spillover intensities associated with the four models we just described. First, the dynamics of the intensity is, in general, similar in the models with and without regressors. We can see that the inclusion of regressors seems to dampen fluctuations in the intensity of the network, on average. That is, when intensity is decreasing, the inclusion of the path factor (black line), for example, decreases the magnitude of the decline. In sum, forward guidance and, to a lesser extent, market uncertainty seem to be relevant drivers of the spillover strength in our network of yield curve surprises.

\section{Monetary Policy and the Yield Curve}

To demonstrate the usefulness of our network approach in understanding how different dimensions of monetary policy propagate across maturities and how they interact with each other, we calculate spatial responses. Since we model the contemporaneous relations between interest rates surprises at a monthly frequency, spatial responses in our setup can be thought of as the evolution of spillovers along the rounds or loops of feedback in a given month. We can then look at how, for example, short-term, medium-term, and long-run surprises or combinations thereof propagate across maturities. In particular, one can think of surprises in the three-month T-bill rate as conventional monetary policy, while surprises in the medium- and long-term bond yields may reflect policies such as quantitative easing or direct interest rate targeting. 


\subsection{Monetary Policy Spillovers}

We start by looking at how various monetary policies, expressed by interest rate surprises at various maturities, propagate through the yield curve. Arguably, this may help our understanding of the effectiveness of a given monetary policy tool for interest rates of particular maturity relevant to the economy at a given point in time.

Since our proposed model is nonlinear, the shape of spatial responses varies with the network intensity as well as with the size of the initial shock. To showcase this, we construct a few examples, where we obtain the spatial responses of interest rate surprises by keeping the size of the shock fixed and varying the network intensity/spatial dependence $\left(\rho_{t}\right)$. We, then, assess the role of the magnitude of the initial surprise (shock) for the spillovers.

Specifically, we assess how a 100 basis points (bps) monetary policy shock, i.e., a 100bps change in an interest rate surprise for each maturity, spills over to the surprises of other maturities. Since the network intensity is time-varying, in principle, we could calculate spatial responses for each month in our sample. For simplicity, we consider spatial responses based on the average, maximum, and minimum network intensity over our sample. This facilitates our discussion on how the network intensity influences the spillovers.

Figure 9 plots the spatial responses based on the average network intensity (0.87). Each panel corresponds to a different shock origin. For example, the first panel, titled " $3 \mathrm{~m}$ Origin," plots the spatial responses to a 100bps increase in the three-month T-bill surprise, i.e., an unexpected increase in the three-month T-bill rate. Green dashed and solid lines represent the responses of surprises in the three-month and six-month T-bill rate, respectively. Blue lines correspond to the responses of the surprises in the one-year (dashed), two-year (solid), and five-year (dotted) government bond yields, respectively. The responses of surprises in the 10-year and long-run yields are shown as red dashed and solid lines, respectively.

A few patterns emerge: First, it takes about 20 rounds of feedback before the network

converges. Second, there are limited spillovers from the three-month T-bill surprises to medium- or longer-term maturities. A 100bps unexpected increase in the three-month Tbill rate yields a surprise in the six-month T-bill rate of about 40bps. Spillovers beyond the six-month maturity become increasingly smaller with increasing maturity. Very small spillovers occur at the long end of the yield curve. Third, spillovers from the medium-term surprises are generally stronger for both the short- and longer-term maturities. For example, a 100bps surprise in the two-year yield is associated with increases of around 100bps for the short-term maturities and around 75bps for the long-term maturities. Also, because the connectedness of the two-year yield surprises is strong, the initial surprise of $100 \mathrm{bps}$ is amplified to about 200bps. Finally, spillovers arising from surprises in the 10-year yield 
are strong for the neighboring maturities (five-year and long-run) but more muted for the short-term maturities.

To understand the role of the network intensity, Figure 10 repeats the same exercise but with a smaller network intensity, i.e., we use the sample minimum of 0.59. Two things stand out: First, the network converges faster since it takes less than ten rounds to reach the maximum spillover effect; second, the size of spillovers is smaller but not proportionally so. For example, a 100bps surprise in the two-year yield induces a surprise of about 20 and 10bps for the six-month and three-month rates, respectively. At the same time, the relative order of which maturities are affected the most changes: While with average intensity, a change to the two-year yield surprise affected the three-month T-bill surprise more than the 10-year one; with the minimum intensity, the opposite is true.

Moreover, Figure 11 plots the same spatial responses associated with the maximum network intensity of about 0.96. In this case, the network converges a lot more slowly and the size of spillovers is large. These simple exercises of varying the network intensity highlight how the network intensity can actually affect the whole network structure. This implies that drivers of the network intensity, such as forward guidance, may influence not only the magnitude but also the relative order of spillovers.

Now, we discuss how the spatial responses vary with the size of the initial shock. Figure 12 shows the responses associated with a $25 \mathrm{bps}$ shock and the average network intensity. A smaller-sized shock does not seem to affect the convergence speed of the network much. As is the case for a 100bps shock, it takes about 20 feedback loops to reach maximum spillover effects. Further, a change of $25 \mathrm{bps}$ in interest rate surprises is generally associated with smaller spillovers across maturities. Finally, the size of the intial shock also seems to matter for the relative order of spillover magnitudes. For example, the 10-year bond surprise is affected most by a surprise in the five-year rate, while, in the case of a 100bps shock, the effect on the five-year maturity dominated the one on the 10-year maturity. This highlights

how nonlinear the network is: not only in terms of the network intensity but also in the size of the initial monetary policy shock.

\subsection{Spillovers of Monetary Policies' Interactions}

Having seen how surprises at various maturities spill over across the yield curve, we now present a few exercises which demonstrate how our framework can be used to understand the interactions of the various dimensions of monetary policies. Our experiments are motivated by the Federal Reserve's Operation Twist, implemented in 2011-2012. Operation Twist is based on the idea that by purchasing longer-term bonds, the Federal Reserve can help drive longer-term yields down, while selling shorter-term bonds at the same time should increase 
the interest rates on the shorter end of the yield curve. In combination, these two actions would "twist" the (typically upward sloping) shape of the yield curve.

For our first exercise, we choose a network intensity of 0.95 , corresponding to the value on September 2011 (start of Operation Twist). We consider two types of shocks at the same time: a positive shock to the three-month T-bill rate of 1 bps and a negative shock to the 10-year bond yield of 5bps. These shocks are calibrated to match the observed daily changes in corresponding bond markets around the Operation Twist announcement on September $21,2011$.

The first panel of Figure 13 plots the spatial responses resulting from a combination of these two shocks. We observe the following: First, given the high network intensity, it takes about 60 iterations for the network to converge, and the size of the spillovers is generally large. Second, and most interestingly, we observe how the two different monetary interventions interplay across the network. That is, initially, the positive three-month Tbill surprise translates into positive surprises for the six-month and one-year yields. At the same time, the negative surprise at the 10-year maturity leads to negative spillovers across the long-run, five-year, and two-year maturities. However, after a few rounds of feedback, the responses at the shorter end of the yield curve begin to turn negative, first for the surprises at the one-year maturity and then for the surprises at the six-month and three-month maturities. Hence, the initially negative shocks at the longer-term maturities eventually dominate the positive shocks at the shorter-term maturities.

To see how the network intensity may influence these combined responses, we reproduce the same exercise but with a lower intensity of 0.87 (sample average). This is an interesting exercise since the central bank may influence the intensity by changing its forward guidance. The second panel of Figure 13 shows that responses to the Operation Twist shocks are generally weaker in the case of lower intensity. In contrast to the case of a higher network intensity, the resulting positive surprises for the short-term maturities do not turn negative. Nevertheless, they are still offset since the spatial responses for the short-term maturities eventually become zero.

Next, we assess how spatial responses to Operation Twist change when we consider a differently sized shock (keeping the network intensity at 0.95; see Panel 3 of Figure 13). In particular, we are interested in how big the change in the three-month T-bill surprise needs to be to maintain a positive response at the short maturities. It would need a 125bps increase in the three-month T-bill surprise to keep its response positive. Nevertheless, the negative spillovers from the long-term maturities (due to the 10-year bond surprise of -5bps) still dampen the initial shock of $125 \mathrm{bps}$ to about $25 \mathrm{bps}$, and spillovers to the six-month T-bill surprise turn negative after about 10 feedback rounds. 
The former exercises demonstrate that pass-through from the three-month surprises to medium- or long-term maturities is rather weak. This leads to negative surprises in the 10year bond yields offsetting or dominating the positive effects at the short-end of the yield curve. As a final exercise, we show how spillovers evolve when there is 1 bps shock to the six-month T-bill or the two-year yields instead. Panel 4 of Figure 13 shows that a positive surprise in the six-month T-bill has positive spillover effects for the three-month and oneyear maturities. The two-year maturity is barely affected, as positive and negative spillovers from the short and long end of the yield curve eventually cancel each other. Moreover, the negative surprise in the 10-year yields leads to negative responses for the long-run and five-year maturities. If we consider a positive surprise in the two-year government bond yield instead, we see positive spillovers to maturities of two years and under. Interestingly, spillovers to the three-month T-bill surprise are smaller than in the case of a positive surprise to the six-month T-bill rate.

These exercises are just a few illustrative and hypothetical scenarios of how the proposed framework can help our understanding of the interactions between different dimensions of monetary policy. A few key implications for monetary policy emerge: First, pass-through from the short-term maturities to medium- and long-term maturities is muted. If the only intention of the central bank were to affect the short-term interest rates, yet keep the yields along the rest of yield curve mostly unaffected, short-term interest rate targeting would support that objective. However, since surprises at the medium-term maturities are well connected to both the short and long end of the yield curve, targeting medium-term bond yields may be the most effective way to move all the yields in the same direction. Second, if the intention of a central bank is to (un)flatten the yield curve or affect a particular segment of the yield curve more relative to others, then there a few alternative ways that the central bank may achieve this - the outcome depends on the targeted maturity as well as the size of the shock (amount of bonds sold/bought). Understanding the spillover structure can help the central bank achieve its objectives more efficiently by changing the relevant interest rates with smaller interventions in the bond market ("more bang for its buck"). Third, supplementing the interest rate targeting policies with adequate forward guidance can make the central bank even more efficient.

\section{Conclusion}

We propose a dynamic spatial lag model with an unobserved, potentially asymmetric weight matrix and a time-varying, data-driven intensity parameter. We employ the proposed framework to assess spillovers of interest rate surprises across the yield curve over time. 
We find that the network is sparse and that only adjacent maturities are connected directly to each other. Furthermore, we document strong time variation in the intensity parameter, mainly driven by forward guidance. Our results suggest significant and nontrivial complementaries between the various dimensions of monetary policy. We illustrate how understanding the spillover structure can be useful for monetary policy makers.

Though arguably, our paper addresses the first link in the transmission of monetary policy, i.e., the spillovers of the surprises across the yield curve, one could certainly nest our setup into more traditional empirical monetary models to assess the effects of the various dimensions of monetary policy on the economy at large. This could be done with further methodological extensions to allow for shrinkage in order to deal with the increase in the parameter space effectively. 


\section{References}

Anselin, L. (1988). Spatial econometrics: methods and models. Studies in Operational Regional Science. Springer.

Bailey, N., Holly, S., and Pesaran, M. H. (2016). A two-stage approach to spatio-temporal analysis with strong and weak cross-sectional dependence. Journal of Applied Econometrics, 31(1):249-280.

Bhattacharjee, A. and Jensen-Butler, C. (2013). Estimation of the spatial weights matrix under structural constraints. Regional Science and Urban Economics, 43(4):617-634.

Blasques, F., Koopman, S. J., Lucas, A., and Schaumburg, J. (2016). Spillover dynamics for systemic risk measurement using spatial financial time series models. Journal of Econometrics, 195(2):211-223.

Bu, C., Rogers, J., and Wu, W. (2019). A unified measure of Fed monetary policy shocks. Finance and Economics Discussion Series 2019-043, Board of Governors of the Federal Reserve System (U.S.).

Campbell, J., Evans, C., Fisher, J., and Justiniano, A. (2012). Macroeconomic effects of Federal Reserve forward guidance. Brookings Papers on Economic Activity, 43(1):1-80.

Catania, L. and Billé, A. G. (2017). Dynamic spatial autoregressive models with autoregressive and heteroskedastic disturbances. Journal of Applied Econometrics, 6(32):11781196.

Chun, A. L. (2011). Expectations, bond yields, and monetary policy. Review of Financial Studies, 24(1):208-247.

Creal, D., Koopman, S. J., and Lucas, A. (2011). A dynamic multivariate heavy-tailed model for time-varying volatilities and correlations. Journal of Business and Economic Statistics, 29(4):552-563.

Creal, D., Koopman, S. J., and Lucas, A. (2013). Generalized autoregressive score models with applications. Journal of Applied Econometrics, 28:777-795.

Creal, D., Schwaab, B., Koopman, S. J., and Lucas, A. (2014). Observation driven mixedmeasurement dynamic factor models. Review of Economics and Statistics, 96:898-915.

Dovern, J., Fritsche, U., and Slacalek, J. (2012). Disagreement among forecasters in G7 countries. The Review of Economics and Statistics, 94(4):1081-1096. 
Ganics, G., Rossi, B., and Sekhposyan, T. (2019). From fixed-event to fixed-horizon density forecasts: Obtaining measures of multi-horizon uncertainty from survey density forecasts. Mimeo.

Gardner, B., Scotti, C., and Clara, V. (2020). Words speak as loudly as actions: The response of equity prices to macroeconomic announcement. Mimeo.

Gürkaynak, R. S., Sack, B., and Swanson, E. (2005). Do actions speak louder than words? The response of asset prices to monetary policy actions and statements. International Journal of Central Banking, 1(1).

Harvey, A. and Luati, A. (2014). Filtering with heavy tails. Journal of the American Statistical Association, 109(507):1112-1122.

Harvey, A. C. (2013). Dynamic models for volatility and heavy tails, with applications to financial and economic time series. Number 52. Cambridge University Press.

Inoue, A. and Rossi, B. (2019). The Effects of Conventional and Unconventional Monetary Policy: A New Approach. Working Papers 1082, Barcelona Graduate School of Economics.

Knüppel, M. and Vladu, A. (2016). Approximating fixed-horizon forecasts using fixed-event forecasts. Deutsche Bundesbank Discussion Paper, 28.

Koopman, S. J., Lucas, A., and Scharth, M. (2016). Predicting time-varying parameters with parameter-driven and observation-driven models. Review of Economics and Statistics, 98(1):97-110.

Kuttner, K. N. (2001). Monetary policy surprises and interest rates: Evidence from the Fed Funds futures market. Journal of Monetary Economics, 47(3):523-44.

Lam, C. and Souza, P. C. (2016). Detection and estimation of block structure in spatial weight matrix. Econometric Reviews, 35(8-10):1347-1376.

Lam, C. and Souza, P. C. (2020). Estimation and selection of spatial weight matrix in a spatial lag model. Journal of Business \& Economic Statistics, 38(3):693-710.

Tonzer, L. (2015). Cross-border interbank networks, banking risk and contagion. Journal of Financial Stability, 18:19-32.

Wang, D., van Lelyveld, I., and Schaumburg, J. (2018). Do information contagion and business model similarities explain bank credit risk commonalities? DNB Working Papers 619, Netherlands Central Bank, Research Department. 
Tables and Figures 


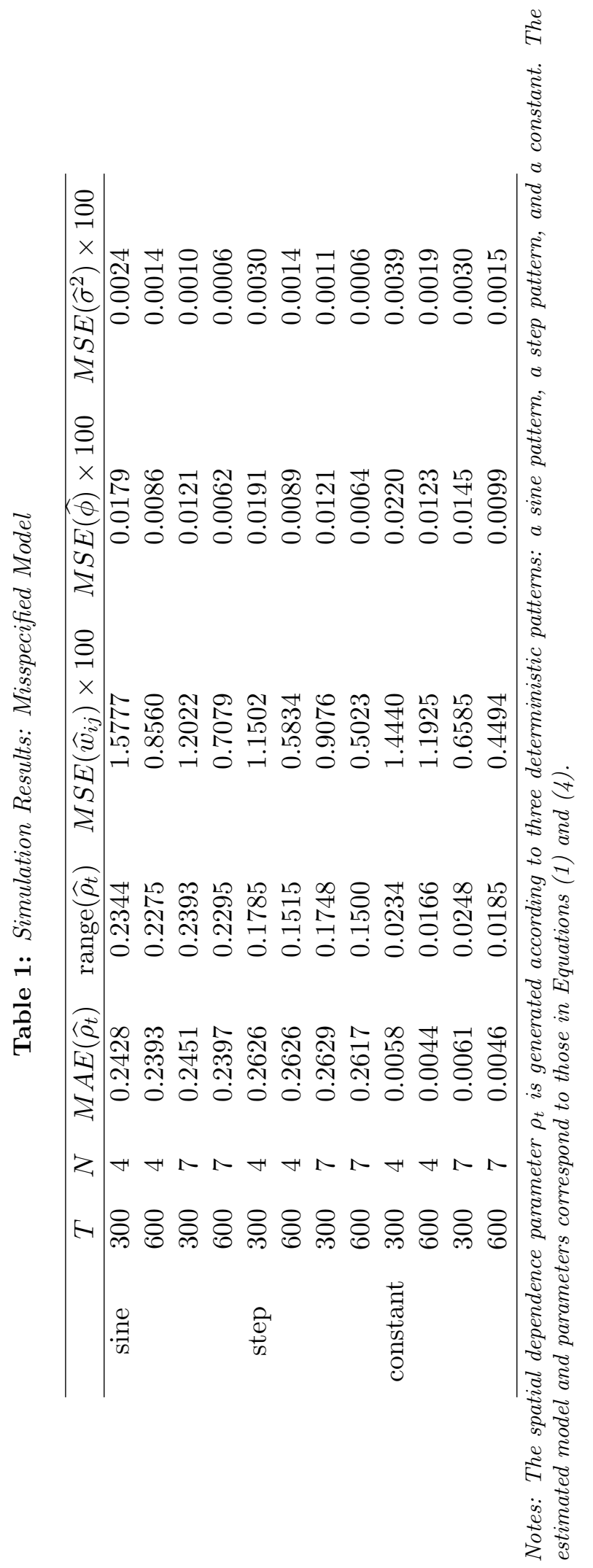




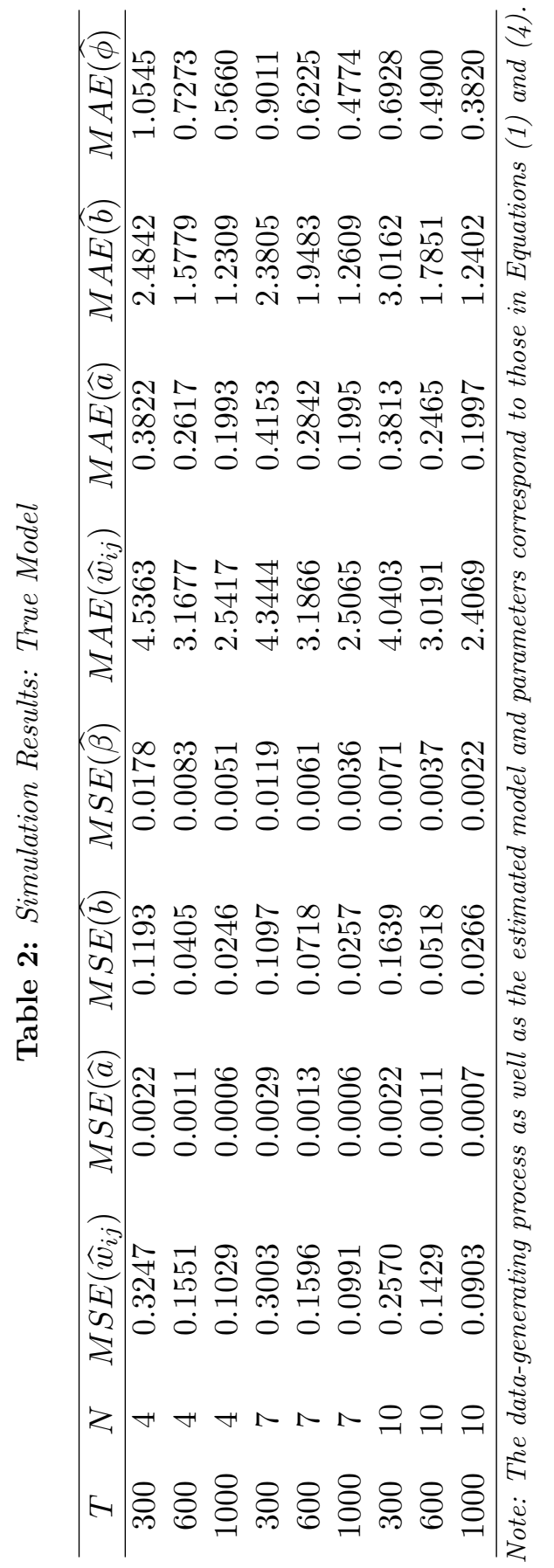


Table 3: Drivers of Network Dynamics: Estimated Model Specifications

\begin{tabular}{c|crrr}
\hline \hline & Model 1 & Model 2 & Model 3 & Model 4 \\
\hline $\mathbf{a}$ & 0.10 & 0.07 & 0.08 & 0.07 \\
$\mathbf{b}$ & 0.90 & 0.94 & 0.87 & 0.92 \\
\hline Path & & -0.50 & -0.39 & -0.49 \\
VIX & & & 0.02 & 0.01 \\
IP & & & & -1.47 \\
\hline AIC & -916.31 & -919.81 & -918.27 & -915.71
\end{tabular}

Note: The table shows parameter estimates associated with the law of motion for the network dynamics, i.e., $f_{t+1}$ in Equation (4). 
Figure 1: Tracking the Spatial Dependence Parameter $\left(\rho_{t}\right)$

(a) Tracking performance, $T=300, N=7$.
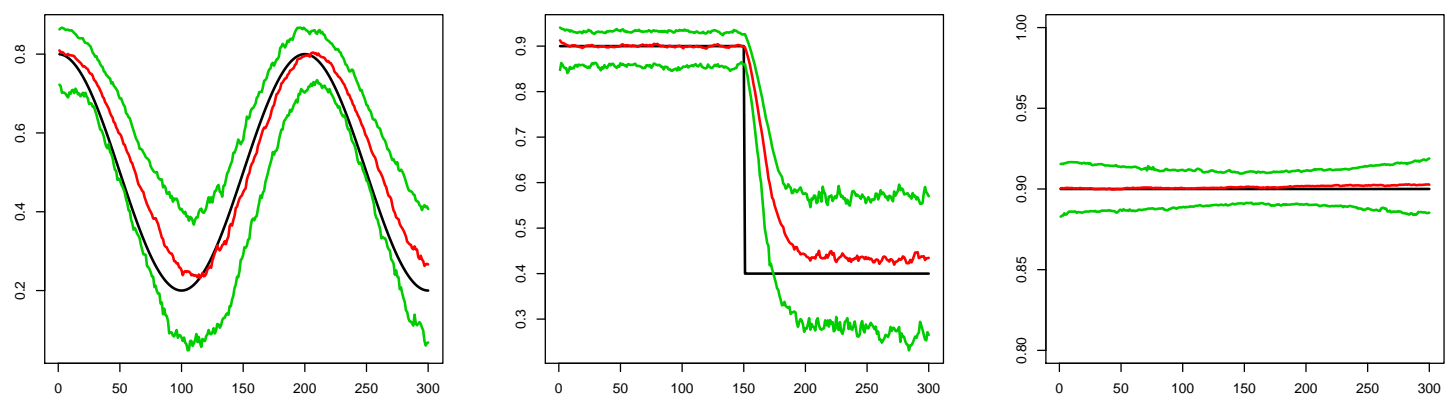

(b) Tracking performance, $T=600, N=7$.
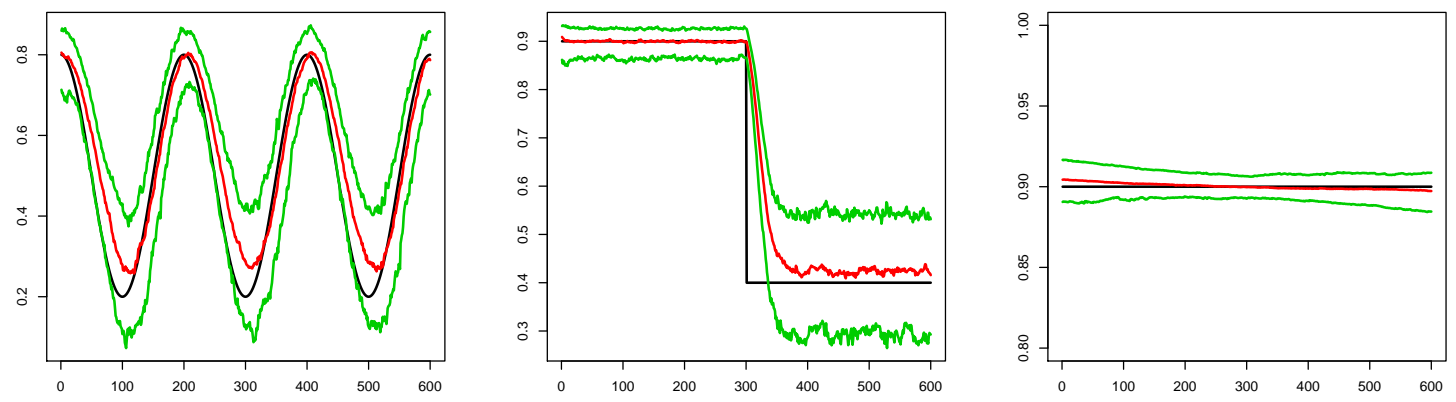

Notes: The spatial dependence parameter $\rho_{t}$ is generated according to three deterministic patterns: a sine pattern (first column), a step pattern (second column), and a constant (third column). The estimated model and parameters correspond to those in Equations (1) and (4). 
Figure 2: Kernel Densities of Estimated Parameters, $T=300,600,1000, N=4$.
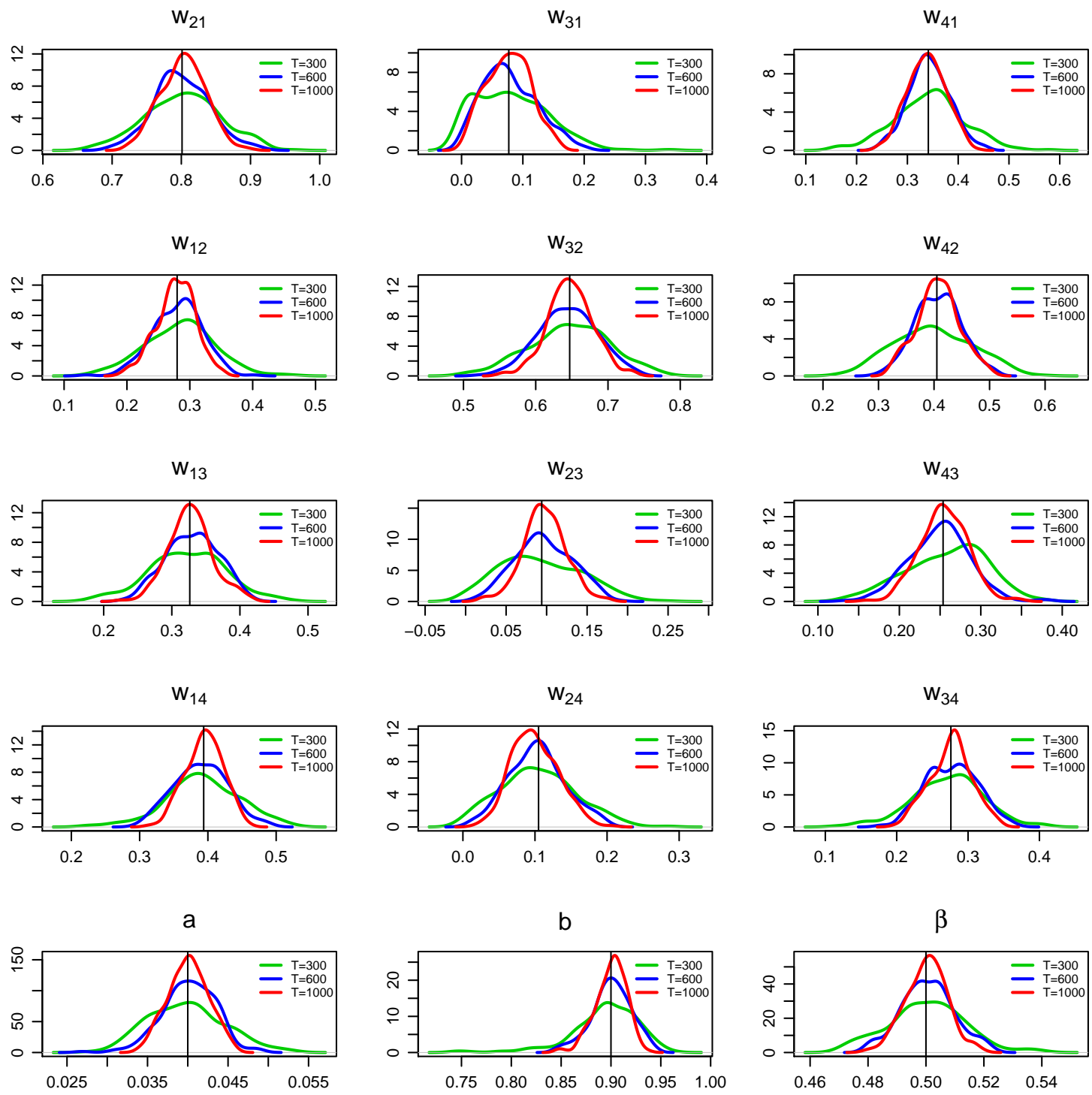

Notes: The data-generating process as well as the estimated model and parameters correspond to those in Equations (1) and (4). The distributions are smoothed with a Gaussian kernel estimation. 
Figure 3: Data: Interest Rate Surprises across Various Maturities
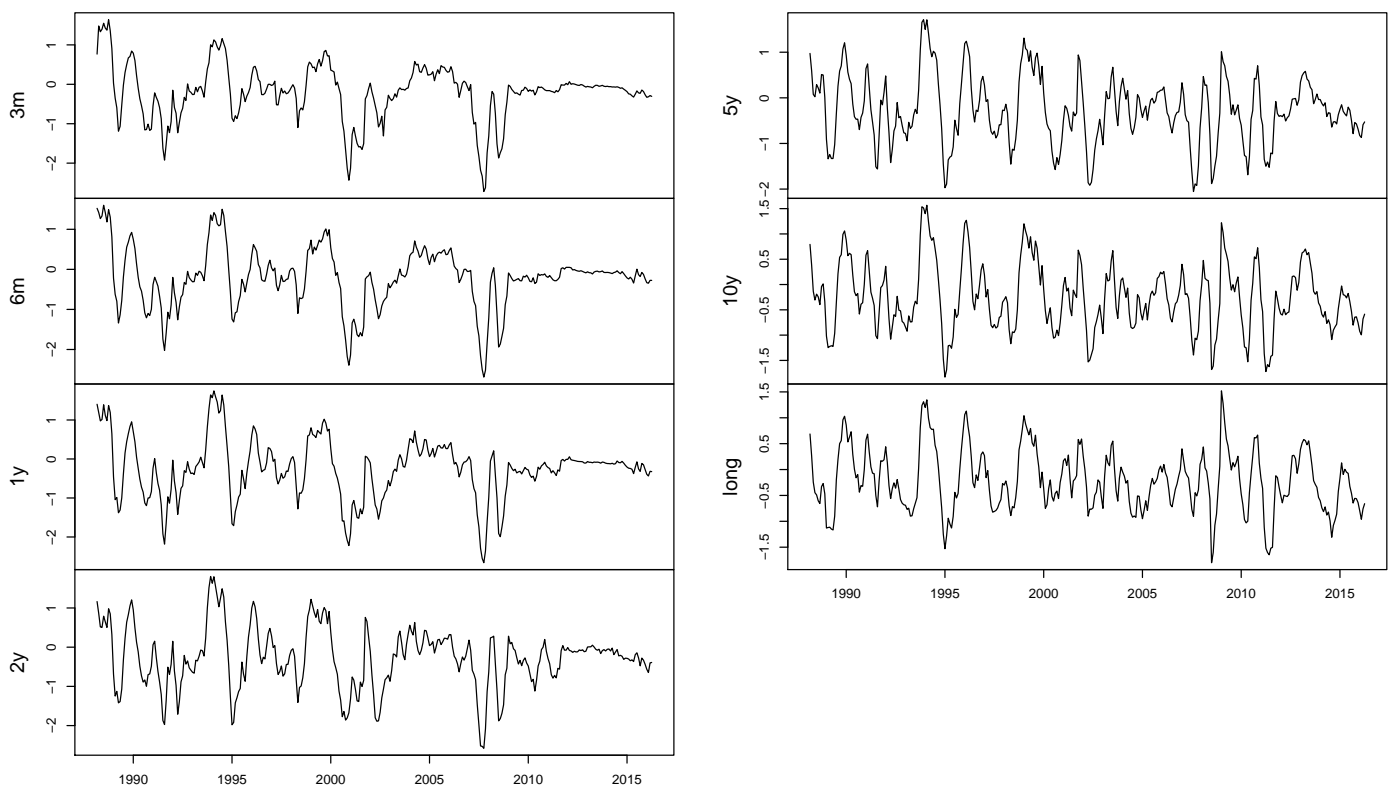

Notes: Surprises are calculated as the differences between the realization of the US government bond yields and expectations from the BCFF at $h=6$ forecast horizon for seven maturities (indicated on the y-axis). The sample ranges from March 1988 until April 2016.

Figure 4: Data: Changes in Government Bond Yields across Various Maturities.
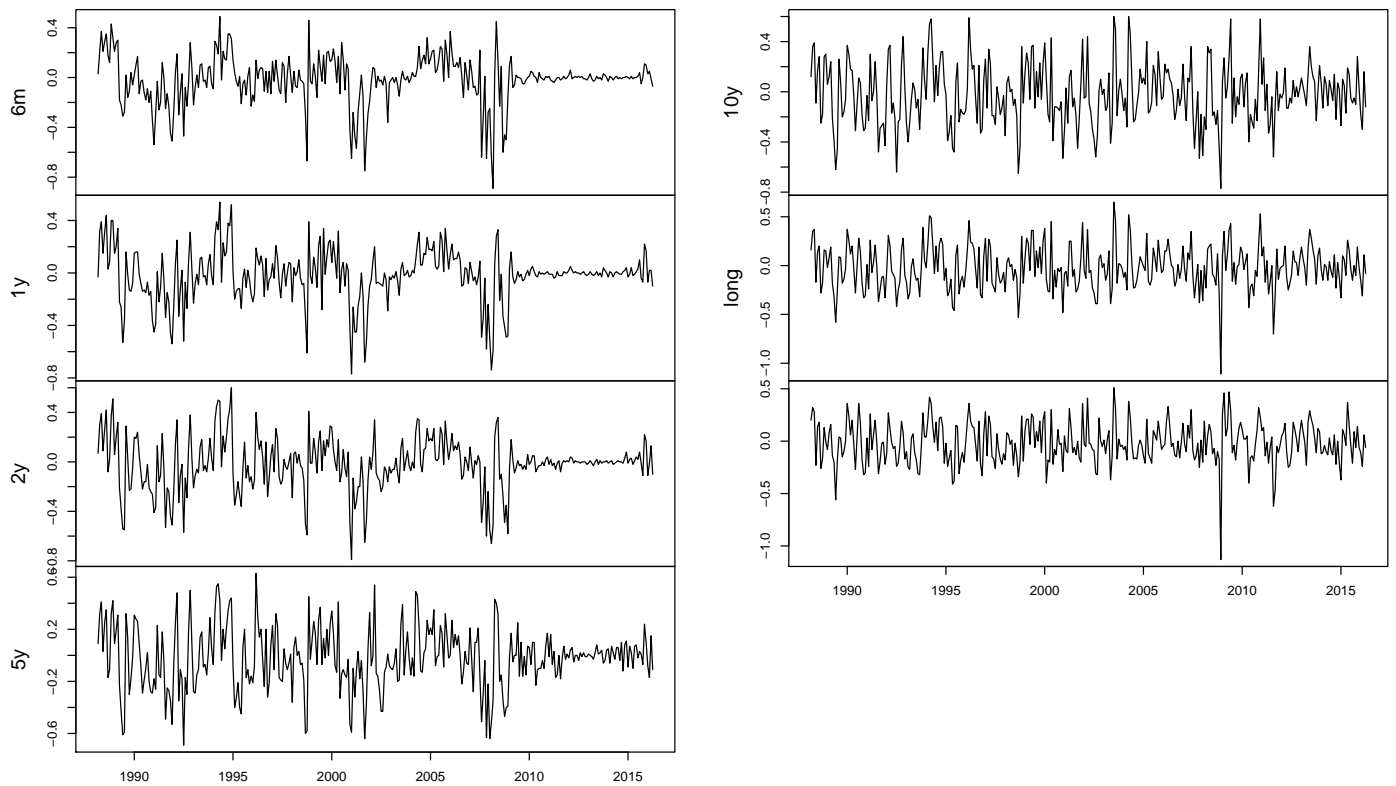

Notes: Changes in government bond yields are used as control variables, in our context yielding identification. The sample ranges from March 1988 until April 2016. 
Figure 5: Dynamic Network of Yield Curve Surprises

(a) Estimated Weights Matrix, W

\begin{tabular}{c|ccccccc} 
& $\mathbf{3 m}$ & $\mathbf{6 m}$ & $\mathbf{1 y}$ & $\mathbf{2 y}$ & $\mathbf{5 y}$ & $\mathbf{1 0 y}$ & $\mathbf{L R}$ \\
\hline $\mathbf{3 m}$ & 0 & 1.00 & 0 & 0 & 0 & 0 & 0 \\
$\mathbf{6 m}$ & 0.20 & 0 & 0.80 & 0 & 0 & 0 & 0 \\
$\mathbf{1 y}$ & 0.10 & 0.34 & 0 & 0.56 & 0 & 0 & 0 \\
$\mathbf{2 y}$ & 0 & 0 & 0.58 & 0 & 0.42 & 0 & 0 \\
$\mathbf{5 y}$ & 0 & 0 & 0 & 0.43 & 0 & 0.57 & 0 \\
$\mathbf{1 0 y}$ & 0 & 0 & 0 & 0 & 0.63 & 0 & 0.37 \\
$\mathbf{L R}$ & 0 & 0 & 0 & 0 & 0 & 1.00 & 0
\end{tabular}

(b) Network Graph

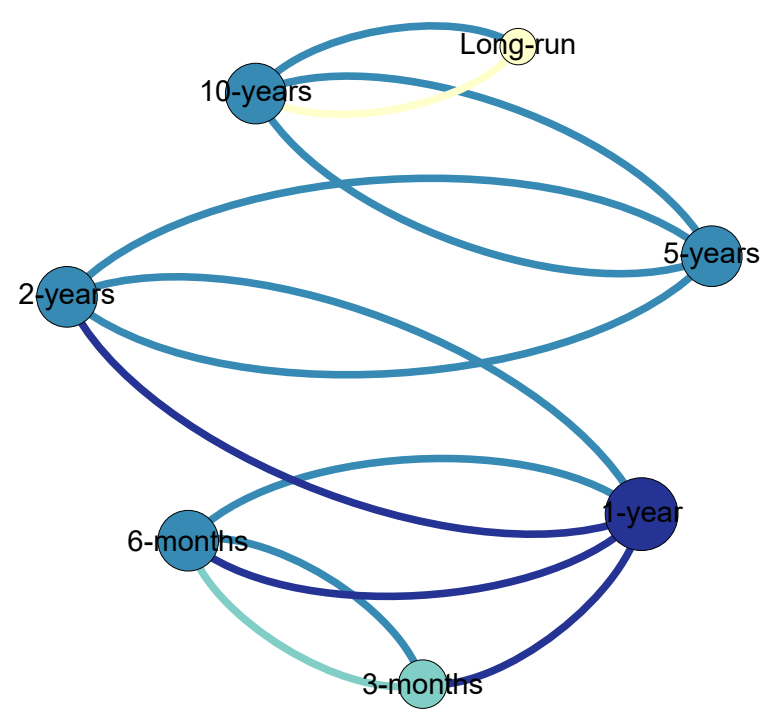

Notes: Panel (a) shows the estimated values of weights matrix, W. Panel (b) visualizes the static network. The bigger and darker-colored nodes represent nodes with a larger number of connections. For instance, the one-year surprise is in a darker blue because it has five connections relative to the surprises in either the two-year, five-year, or 10-year maturities, where each has four connections. Edges have the same color as the node of origin. 
Figure 6: Time-varying Network Intensity

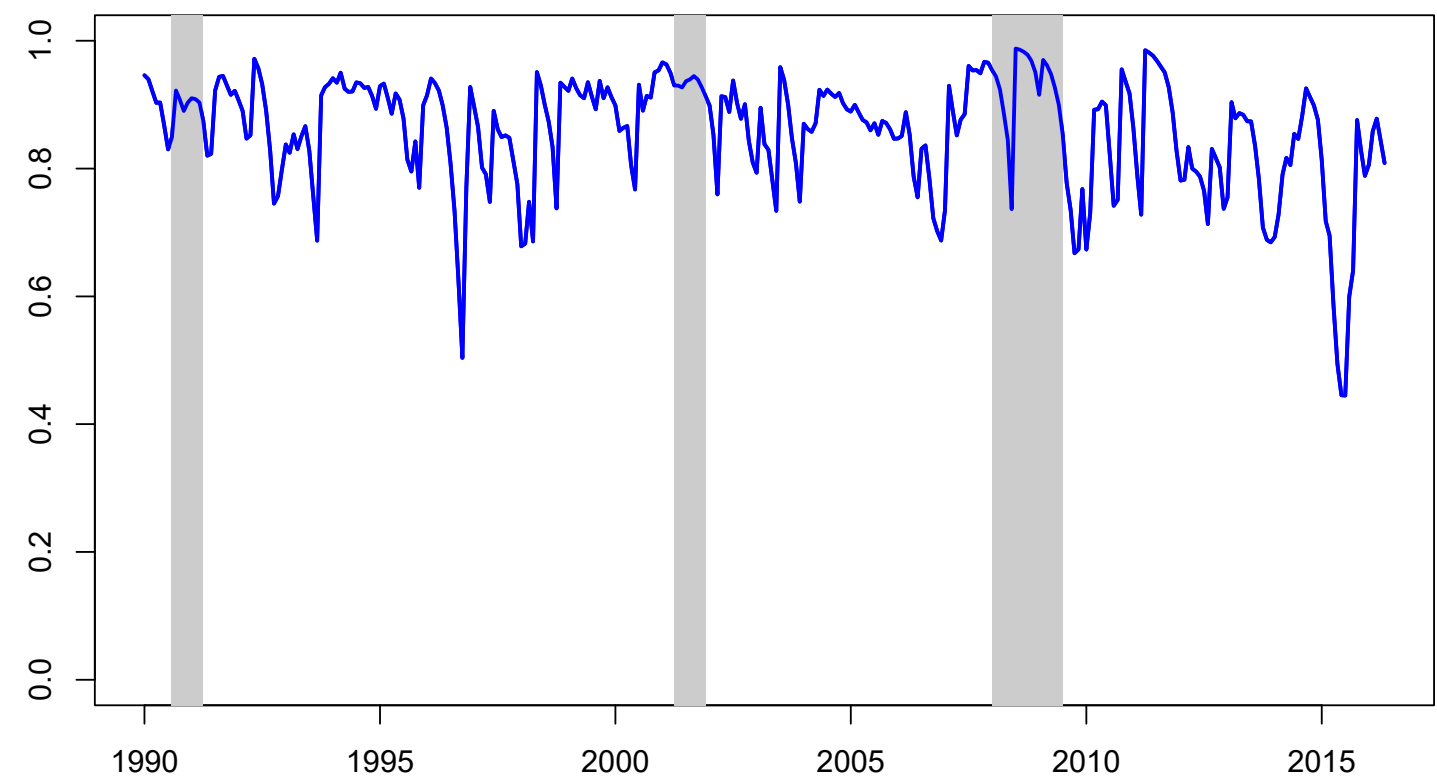

Note: The figure documents the path of network intensity, $\rho_{t}$, in the presence of no exogenous drivers, i.e., when $c=0$.

Figure 7: Data: Drivers of Network Dynamics

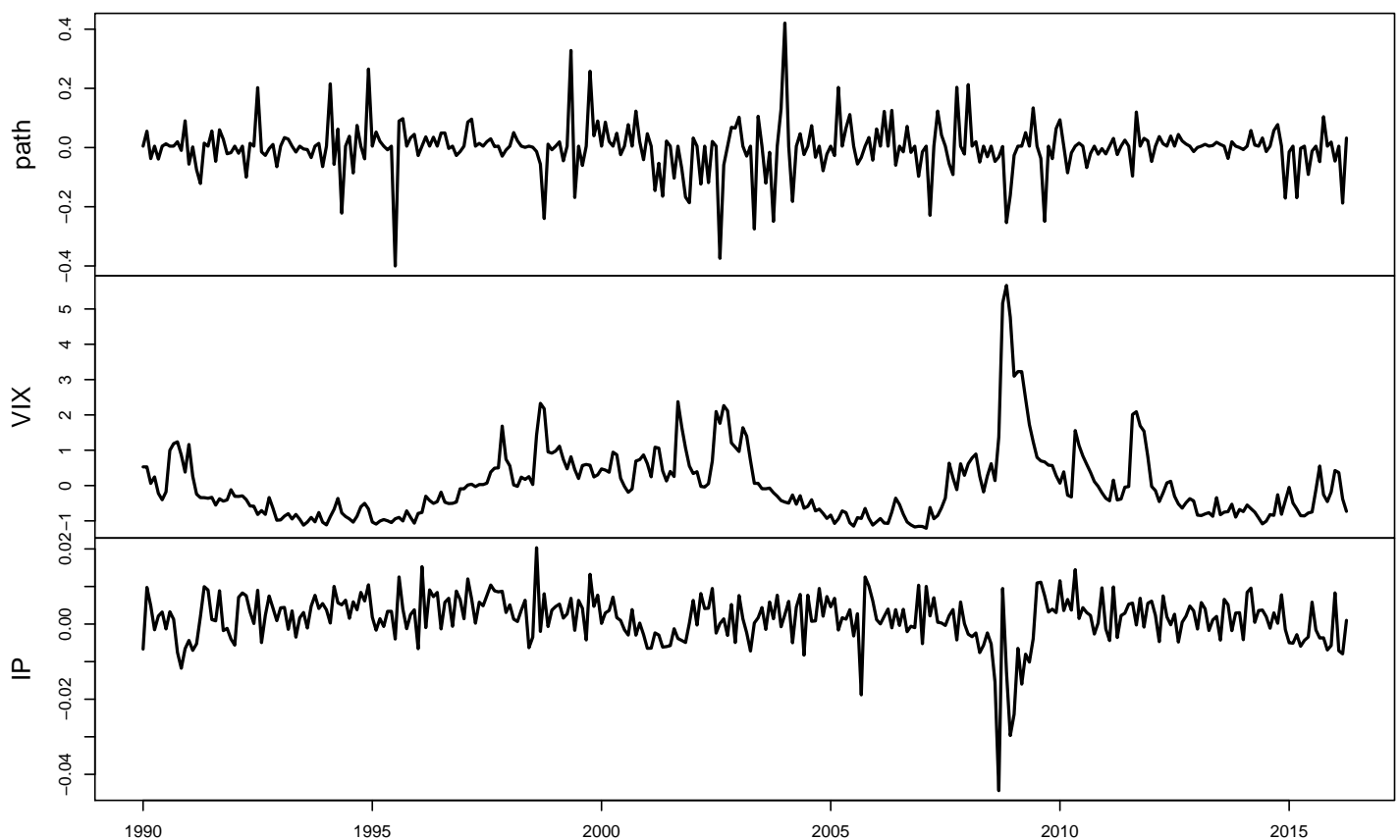

Notes: The figure shows the time series of the path factor extracted from high-frequency interest rate futures, as well as the implied volatility index (VIX) and the industrial production index (IP). The sample ranges from January 1990 until April 2016. 
Figure 8: Time-varying Network Intensity with Covariates

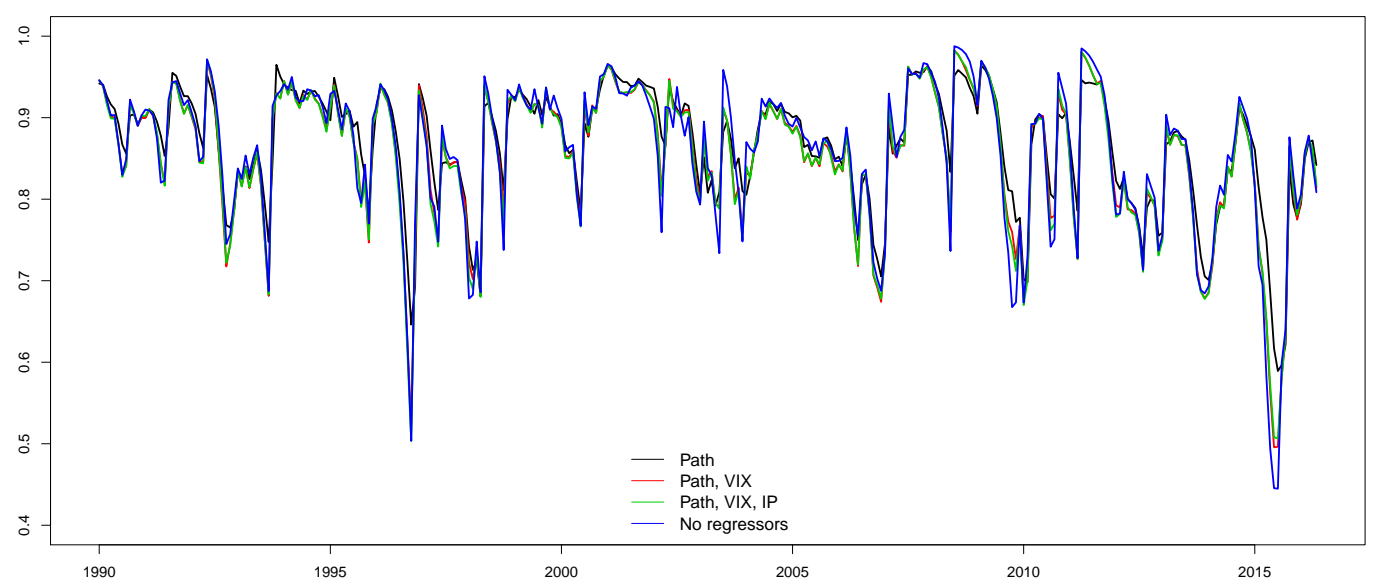

Notes: The figure documents the path of network intensity, $\rho_{t}$, in the presence of various exogenous drivers. In addition, it reports the case with no exogeneous drivers, i.e., when $c=0$, for comparison.

Figure 9: Spatial Responses to 100bps Monetary Policy Shocks: Average Intensity
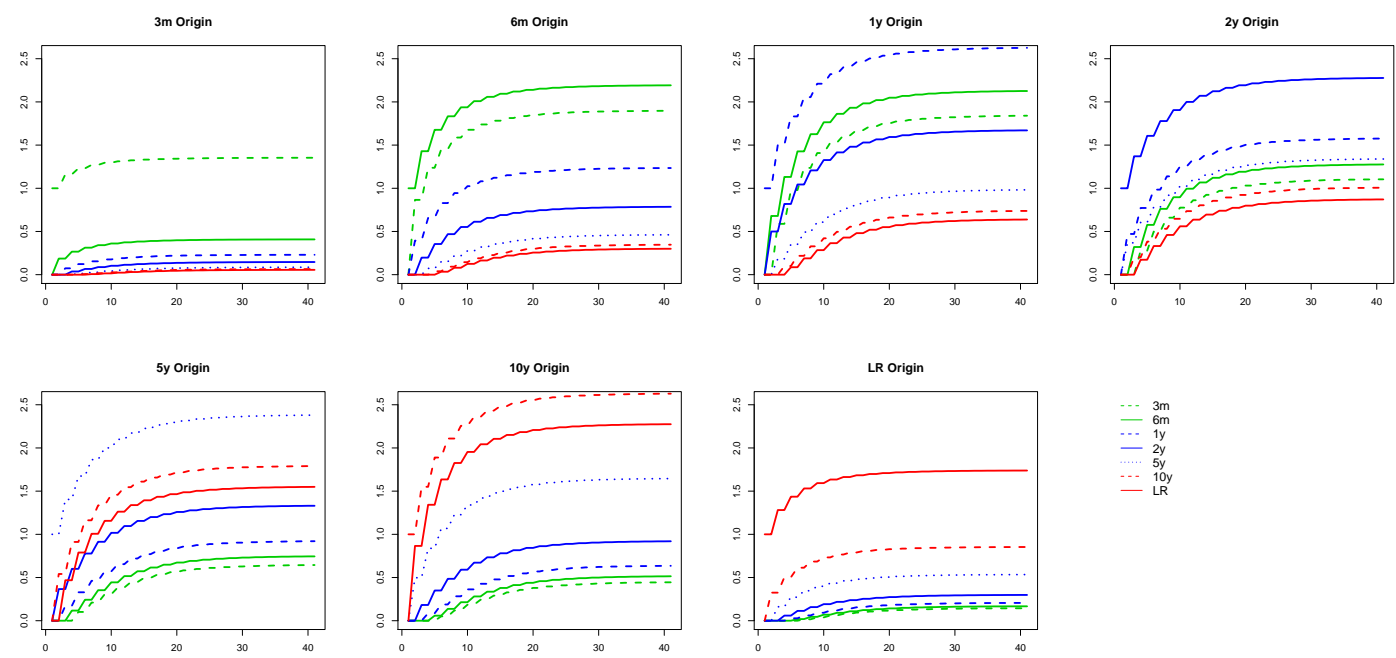

$\begin{array}{cc}- & 3 m \\ - & 6 m \\ \cdots & 1 y \\ - & 2 y \\ -5 y \\ \cdots & 10 y \\ - & \text { LR }\end{array}$

Notes: The estimated model and parameters correspond to those in Equations (1) and (4), where network intensity is driven by the score and the "path" factor. We present the feedback effects based on Equation (3) and the sample average of the network intensity parameter $\rho_{t}$. 
Figure 10: Spatial Responses to 100bps Monetary Policy Shocks: Minimum Intensity
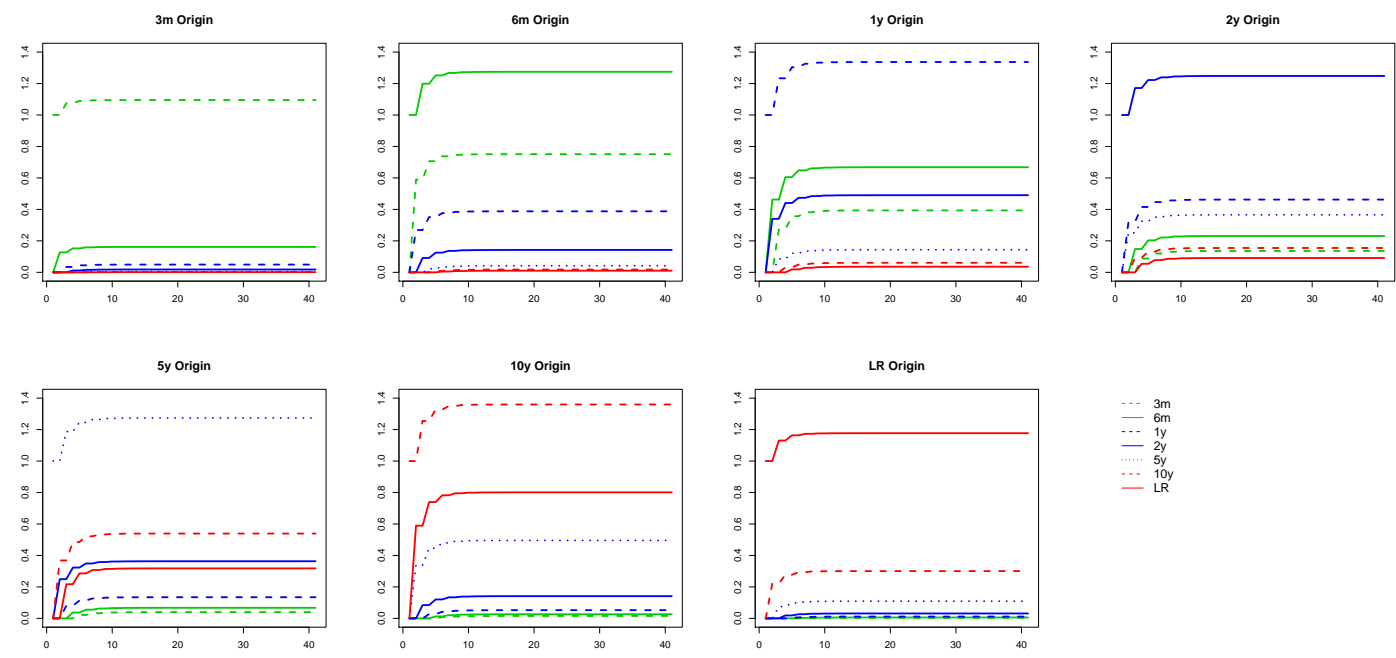

Notes: The estimated model and parameters correspond to those in Equations (1) and (4), where network intensity is driven by the score and the "path" factor. We present the feedback effects based on Equation (3) and the minimum value of the sample network intensity parameter $\rho_{t}$.

Figure 11: Spatial Responses to 100bps Monetary Policy Shocks: Maximum Intensity
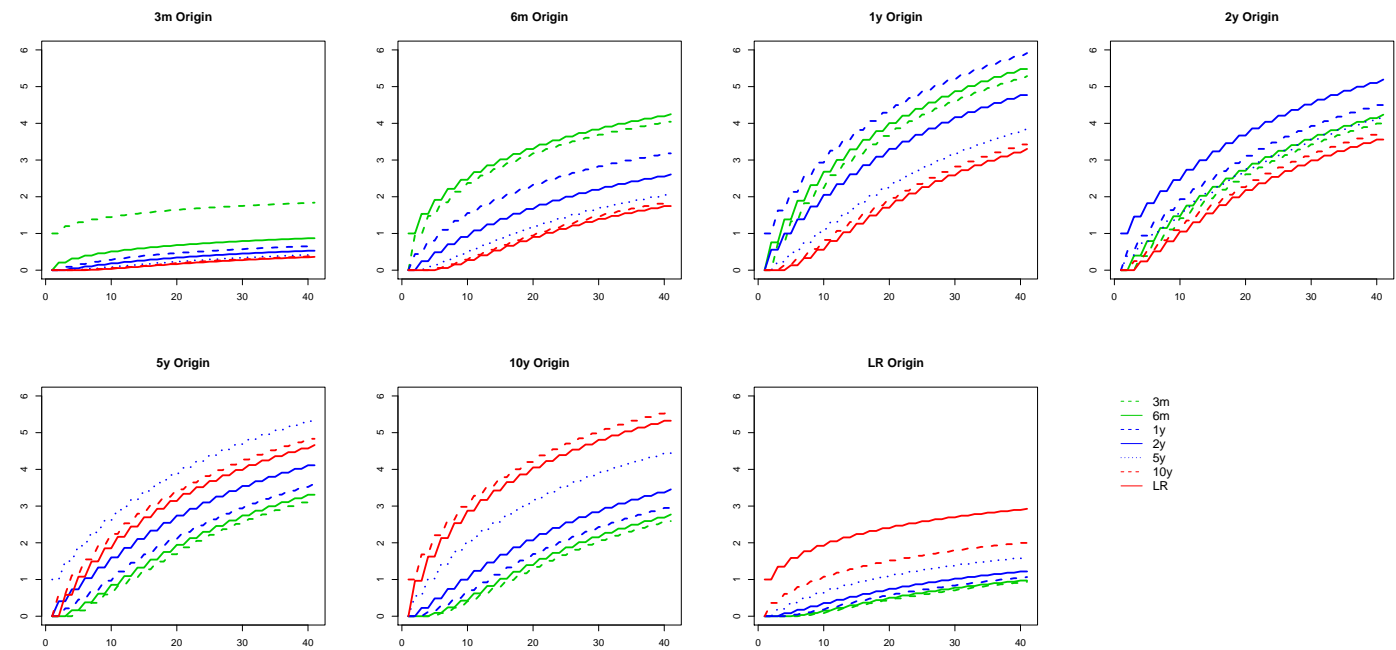

\begin{tabular}{cc} 
& $3 m$ \\
- & $6 m$ \\
$\cdots$ & $1 y$ \\
- & $2 y$ \\
\hdashline$-5 y$ \\
$\cdots$ & $10 y$ \\
- & $L R$
\end{tabular}

Notes: The estimated model and parameters correspond to those in Equations (1) and (4), where network intensity is driven by the score and the "path" factor. We present the feedback effects based on Equation (3) and the maximum value of the sample network intensity parameter $\rho_{t}$. 
Figure 12: Spatial Responses to 25bps Monetary Policy Shocks
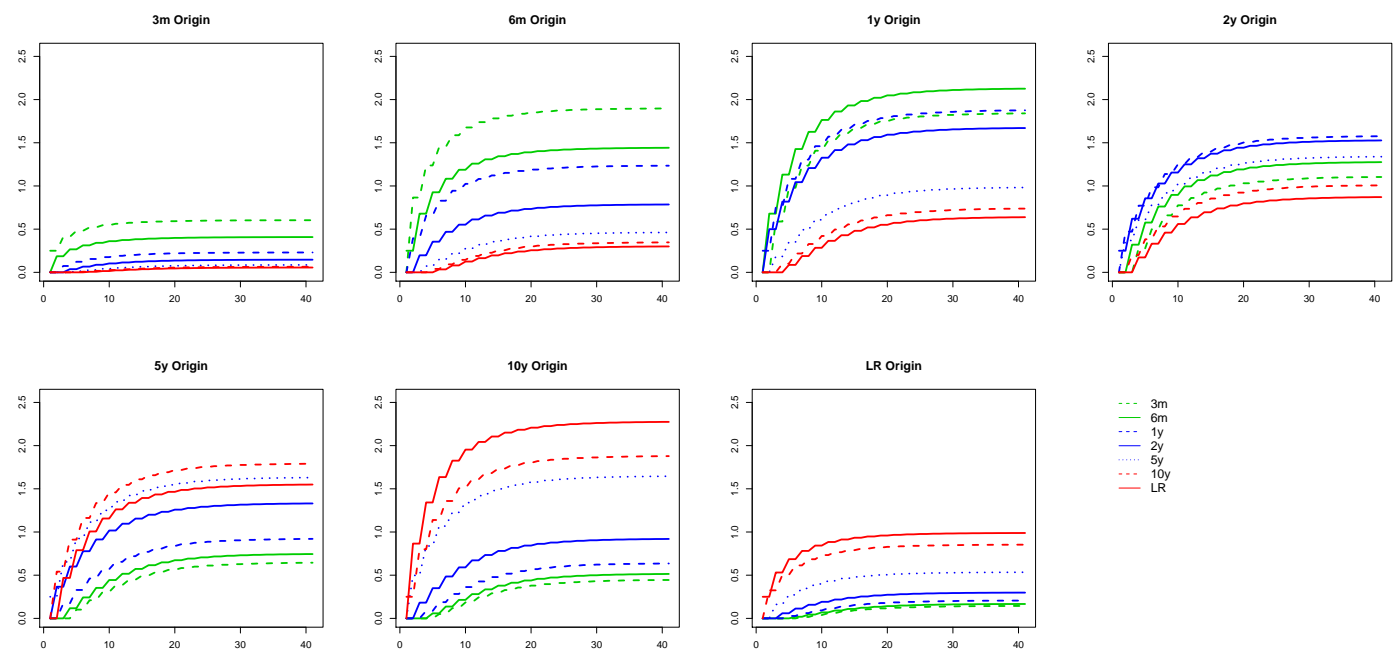

$\begin{array}{cc} & 3 m \\ - & 6 m \\ \cdots & 1 y \\ - & 2 y \\ -2 y \\ \cdots-5 y \\ -10 y \\ - & \text { LR }\end{array}$

Notes: The estimated model and parameters correspond to those in Equations (1) and (4), where network intensity is driven by the score and the "path" factor. We present the feedback effects based on Equation (3) and the mean value of the sample network intensity parameter $\rho_{t}$.

Figure 13: Spatial Responses to Monetary Policies' Interactions
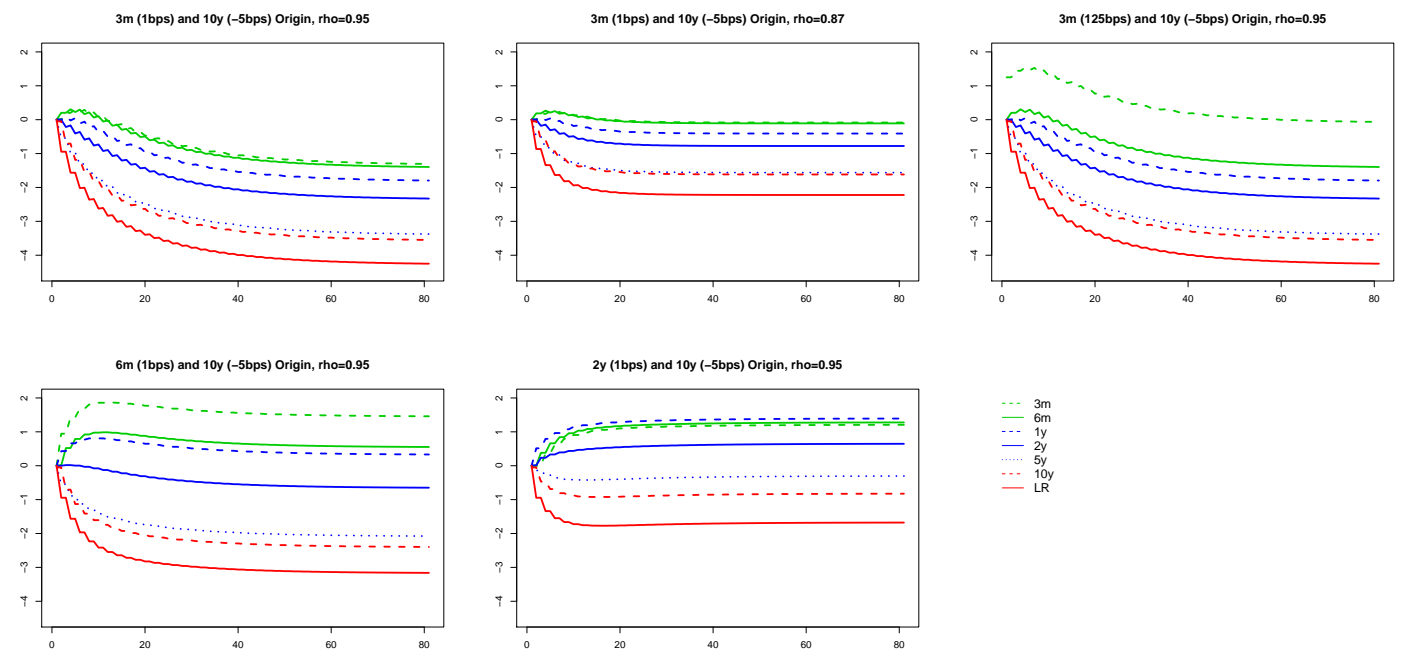

Notes: The estimated model and parameters correspond to those in Equations (1) and (4), where network intensity is driven by the score and the "path" factor. We present the feedback effects based on Equation (3) and the sample network intensity parameter $\rho_{t}=0.95$ as during the Operation Twist episode. 